\title{
Computer tailored intervention for older smokers using transdermal nicotine
}

\author{
C Tracy Orleans, Neal R Boyd, Elizabeth Noll, Linda Crosette, Bernard Glassman
}

This paper presents inital outcomes of a randomised controlled trial evaluating the efficacy of a self help intervention combining a quit smoking guide designed for older adults with a series of personalised computer tailored cessation messages. These messages were delivered through a state prescription plan, similar to the prescription and pharmacy benefit management plans widely used by managed care organisations to control pharmacy costs and improve appropriate drug use. Prescription plans offer a channel which is especially appropriate for older adults since they consume a disproportionate share of medications. ${ }^{1}$

A previous study showed that the Clear Horizons guide, ${ }^{2}$ targeted to the special quitting needs and barriers of older adults, was more effective than a generic quitting guide, and that brief phone calls offering individualised advice further improved the efficacy of the guide. ${ }^{3}$ Since tailored mailings represent a potentially lower cost tailoring strategy than proactive phone counselling, the aim of this study was to evaluate the efficacy of the Clear Horizons guide paired with the same kind of personalised advice delivered through individually tailored computer generated quitting letters.

To test the efficacy of this combined targeted and tailored intervention, 470 smokers aged 65 years and older who filled a transdermal nicotine prescription through Pennsylvania Pharmaceutical Assistance Contract for the Elderly were randomly assigned to receive either usual care (a fact sheet on patch assisted quitting) or a copy of the tailored Clear Horizons guide for older smokers and a series of seven personalised computer generated mailings. Tailored messages were based on past research identifying the factors associated with general quitting success and with patch assisted quitting among older smokers. ${ }^{45}$ Verbal consent for this study was obtained at the time of a baseline interview. Only $4 \%$ of eligible subjects declined. The first mailing was sent out an average of 10 days after subjects filled their prescriptions; the remainder were mailed over a six month period. Both the messages and graphics in these mailings were tailored.
Subjects were mostly female, white, and had not completed high school. With a mean age of 72 years, they had smoked on average for over half a century. They currently smoked a mean of 22 cigarettes a day, and $75 \%$ said they smoked within 30 minutes of awakening-a sign of high nicotine addiction.

Follow up phone interviews were conducted at six and 12 months post-baseline. Six month follow up ( $86 \%$ response rate) showed that the treatment group subjects were more likely than control group subjects to make at least one serious quit attempt $(93 \% \vee 84 \%)$ and to report seven day point prevalence abstinence $(40 \%$ v 33\%) ( $\mathrm{p}<0.05)$. About half of the treatment subjects said they had read all or most of the guide and the mailings. At 12 months ( $78 \%$ response rate), subjects in the tailored treatment group still reported significantly more quit attempts $(88 \%$ v $82 \%$; $\mathrm{p}<0.05$ ), but group differences in quit rates were no longer significant $(33 \% v 31 \%)$. This may reflect a weakening in the effect of the mailings sent during the first six months. Analyses are now underway examining quit rates for treatment group subjects who did and did not read the targeted and tailored self help materials, predicting stronger treatment effects for those who did. Results show promise for integrating computer tailored messages into the treatment of older adult smokers.

This research was supported by NHLBI grant \#RO 1-HL-S0489.

1 Rimer BK, Orleans CT, Keintz MI, et al. The older smoker: status, challenges and opportunities for intervention. Chest 1990;97:547-53.

2 Orleans CT, Rimer BK, Fleisher L, et al. Clear Horizons: a quit smoking guide for smokers aged 50 and older. Philadelphia: Fox Chase Cancer Center, 1995.

3 Rimer BK, Orleans CT, Fleisher L, et al. Does tailoring matter? The impact of a tailored guide on ratings and short-term smoking-related outcomes. Health Educ Res 1994;9:69-84.

4 Orleans CT. Reducing tobacco harms among older adults: a critical agenda for tobacco control. Tobacco Control 1997;6:161-3.

5 Orleans CT, Resch N, Noll E, et al. Use of transdermal nicotine in a state level prescription plan for the elderly: a first look at real-world patch users. $\mathcal{F} A M A$ 1994;271:601-7. 